\title{
Investigation of irradiated volume in linac- based brain hypo-fractionated stereotactic radiotherapy
}

Mark Ruschin ${ }^{1,2^{*}}$, Arjun Sahgal ${ }^{1,2}$, Hany Soliman ${ }^{1,2}$, Sten Myrehaug ${ }^{1,2}$, May Tsao ${ }^{1,2}$, Collins Yeboah ${ }^{1,2}$, Arman Sarfehnia, ${ }^{1,2}$, Brige Chugh ${ }^{1,2}$, Alex Kiss $^{3}$ and Young Lee ${ }^{1,2}$

\begin{abstract}
Background: Emerging techniques such as brain hypo-fractionated radiotherapy (HF-RT) involve complex cases with limited guidelines for plan quality and normal tissue tolerances. The purpose of the present study was to statistically parameterize irradiated volume independently of dose prescription, or margin to determine what spread in achievable irradiated volume one may expect for a given case.

Methods: We defined EXT as the total tissue within the external contour of the patient (including the target) and we defined BMP as the contour of the brain minus PTV. Irradiated volumes of EXT and BMP at specific doses (i.e. 50,60\%, etc., of the prescribed dose) were extracted from 135 single-target HF-RT clinical cases, each planned with a single-arc, homogeneous (SAHO) approach in which target maximum dose (Dmax) was constrained to $<130 \%$ of the prescribed dose. Irradiated volumes were subsequently measured for cases involving 2 targets $(N=29), 3$ targets $(N=7)$ and $>3$ targets $(N=10)$ to investigate the effect of target number. We also examined the effect of shape complexity. A series of best fit curves with confidence and prediction intervals were generated for irradiated volume versus total target volume and the resulting model was subsequently validated on a subsequent set of 23 consecutive prospective cases not originally used in curve-fitting. A subset of 30 HF-RT cases were re-planned with a well-published four-arc, heterogeneous (FAHE) radiosurgery planning approach (Dmax could exceed 130\%) to demonstrate how technique affects irradiated volume.
\end{abstract}

Results: For $\mathrm{SAHO}$, strong correlation $\left(\mathrm{R}^{2}>0.98\right)$ was found for predicting irradiated volumes. For a given total target volume, irradiated-volume increased by a range of 1.4-2.9x for $>3$ versus single-targets depending on isodose level. Shape complexity had minor impact on irradiated volume. There was no statistical difference in irradiated volumes between validation and input data $(p>0.2)$. The FAHE-generated irradiated volumes yielded curves and prediction and confidence bands that agreed well with published data indicating that the proposed approach is feasible for cross-institutional comparisons.

Conclusions: A description of irradiated volume for linac-based HF-RT is proposed based on population data. We have demonstrated that the proposed approach is feasible for inter and intra-institutional comparisons.

Keywords: Brain, Hypofractionated, Radiotherapy, Radiosurgery, Metastasis, Irradiated volume

\footnotetext{
* Correspondence: Mark.Ruschin@sunnybrook.ca

${ }^{1}$ Department of Radiation Oncology, Sunnybrook Health Sciences Centre,

Toronto, ON, Canada

${ }^{2}$ Department of Radiation Oncology, University of Toronto, Toronto, ON,

Canada

Full list of author information is available at the end of the article
}

\section{Ciömed Central}

(c) The Author(s). 2017 Open Access This article is distributed under the terms of the Creative Commons Attribution 4.0 International License (http://creativecommons.org/licenses/by/4.0/), which permits unrestricted use, distribution, and reproduction in any medium, provided you give appropriate credit to the original author(s) and the source, provide a link to the Creative Commons license, and indicate if changes were made. The Creative Commons Public Domain Dedication waiver (http://creativecommons.org/publicdomain/zero/1.0/) applies to the data made available in this article, unless otherwise stated. 


\section{Background}

Evidence continues to support the safety and efficacy of stereotactic radiosurgery (SRS) $[1,2]$ and emerging techniques such as hypo-fractionated radiotherapy (HF-RT) [3-5]. For SRS, recent clinical evidence supports the safety in treating up to ten targets in a single fraction [1]. For HF-RT, radiation-induced harm to normal tissue, in particular for large or recurrent disease, is mitigated whilst delivering a clinically effective dose to the target [4-7]. In conjunction with modern treatment planning systems, linear accelerators equipped with high-definition multileaf collimators (MLCs), imageguidance and robotic couches, both SRS and HF-RT are increasing in utility.

Cases are becoming more challenging with centres treating large number of targets, large volumes, recurrent disease, complex shapes, etc. The well-known safety limit of V12 $<10 \mathrm{~cm}^{3}$ for SRS [8-10] may not necessarily apply or be feasible for multiple targets, re-treatment scenarios, or HF-RT. The focus in the literature has deservedly been on investigating what technique offers the best rapid dose fall-off: the more rapid the better [11-13]. However, every paper reports their endpoints in a different way, making interinstitutional comparisons challenging if not impossible. Other approaches involve developing predictive models of dose fall-off [14-16]. The work by Shiraishi et al. develops and validates a knowledge-based approach of an accurate model, however the model itself is hidden to the reader [14]. The approach by Bohoudi et al., on the other hand is tailored specifically towards V12 [16]. Furthermore, there are limited published data specific to HF-RT. In one paper by Ruschin et al., irradiated volume is reported as a function of target volume but only for specific dosefractionation schemes and only for Brain Minus CTV volume, which can be different amongst institutions who apply different PTV margins than those used [17].

In the present study, we propose a framework that all clinics can easily access to evaluate irradiated volume for arbitrary isodose levels; for both untreated brain (Brain Minus PTV) and total tissue; for varying target numbers and shapes. Although irradiated volume depends on many factors such as machine characteristics and technique, we sought to provide a framework through which our institution and others can readily compare population data to each other. The framework can also be applied to prospective individual cases, in which some guidance as to "what is achievable" for that case could be of assistance. Internally, such a framework could provide the basis for treatment plan quality assurance (QA), especially for challenging or off-protocol cases. Additionally, investigating population trends over time can lead to more consistent and higher quality treatment plans. The intention is not to precisely predict irradiated volume for all institutions and techniques, but rather to provide the framework through which irradiate volume can be compared across institutions.

\section{Materials and methods}

Irradiated volume definition

As shown by previous publications, the volume of tissue receiving specific isodose is proportional to the total target volume raised to an exponent $[15,17]$. The primary framework is thus to define irradiated volume for a series of HF-RT patients as [15, 17]:

$$
V_{\text {primary }}(P)=a(P)(P T V)^{b(P)}
$$

where $V_{\text {primary }}(P)$ is volume of the external contour (EXT, entire volume inside the patient surface, including skull, skin, etc.) or Brain-Minus-PTV (BMP, the brain contour minus all target volumes) receiving dose $\mathrm{P}$ (percentage of prescription), PTV is the total target volume, and $a(P)$ and $b(P)$ are empirically determined fitparameters for single, regularly-shaped targets. We use PTV rather than GTV in the present manuscript since PTV margins may vary between centres, which could cause differences in reported Brain-Minus-GTV values for a given target size. Although BMP may have more clinical relevance in terms of correlating with brain toxicity outcomes, EXT is a quantity the all treatment planning systems are capable of producing and in some cases this is the only quantity readily available as it does not depend on a contour of the brain being present. Note that by definition, the BMP volume will always be less than the corresponding EXT volume for the same target volume, since the target volume is included in EXT but subtracted to produce BMP. The framework assumes that the target is covered by at least $95 \%$ and up to $100 \%$ of the prescribed dose, which is common practice in intra-cranial radiotherapy and radiosurgery. The framework also includes the $95 \%$ prediction and confidence intervals (PI95 and CI95), which can be determined using a linear regression model. Note that the PI95 is by definition always larger than the CI95 as it is used to forecast individual measurements, rather than compare populations. The PI95 (rather than the CI95) is taken to be the error term of the fit, as the general application of the framework is to forecast future plans, however where appropriate the CI95 is also used to compare to populations together.

\section{Effect of target number and shape}

In order to improve curve-fitting accuracy, it may be useful to stratify cases according to specific situations. For example, target shape and number have been discussed in the literature as potentially affecting irradiated 
volume [18]. Rather than present Eq. (1) for every possible situation, which could get confusing, our proposed framework is for a centre to first build a model based on single, regularly-shaped targets and then scale that primary model by multiplicative factors. In the present manuscript we examined the effect of number of targets and shape complexity via adjustment factors $F_{N}$ and $R$ as follows.

Adjustment factor for number of targets $-\mathrm{F}_{\mathrm{N}}$

To account for number of targets, we define an adjustment factor $-\mathrm{F}_{\mathrm{N}}(\mathrm{P})$ - as follows:

$$
\left.F_{N}(P)\right) \frac{a-N(P)(P T V)^{b \_N(P)}}{a_{-} 1(P)(P T V)^{b \_1(P)}} \cong \frac{a_{-} N(P)}{a_{-} 1(P)}
$$

where $\mathrm{N}=$ number of targets $(2,3$, or $>3)$, a_1(P), and b_1(P) are the fit parameters for single-targets. Assuming that multiple targets cause a perturbation to the model and in order to reduce the fit to a singleparameter, we intentionally set $\mathrm{b} \_\mathrm{N}(\mathrm{P})$ equal to $\mathrm{b} \_1(\mathrm{P})$. This assumption was tested in a pilot study by comparing a 2-fit operation to a 1-fit operation and we found the overall relative agreement to be within $10 \%$ for total target volumes above $20 \mathrm{~cm}^{3}$. We can thus express irradiated volume for multiple targets the irradiated volume for single-targets times a scalar as follows:

$$
\begin{aligned}
V-N(P) & =F_{N}(P)\left[a_{1(P)}(P T V)^{b 1(P)}\right] \\
& =F_{N}(P) \times V_{\text {primary }}(P)
\end{aligned}
$$

where $V_{\text {primary }}(P)$ is given by Eq. 1 . Note: $F_{N}(P)$ is always $>=1$.

\section{Adjustment factor for shape regularity- $R$}

To account for shape complexity we define a "regularity index" (RI) that relates how spherical a target is: 1.0 being a perfect sphere and values $<1.0$ becoming less spherical [17, 18]. Mathematically, we have defined RI as:

$$
R I=\frac{2 \times V_{S, T}}{V_{T}+V_{S}}=\frac{V_{S, T}}{V_{T}}
$$

where $\mathrm{V}_{\mathrm{T}}$ is the target volume (PTV in the present study), $\mathrm{V}_{\mathrm{S}, \mathrm{T}}$ is the volume of overlap between the target volume and an equivalent-volume sphere positioned at the centre of gravity of the target volume, and $V_{S}$ is the sphere volume, which by definition is equal to $\mathrm{V}_{\mathrm{T}}$. Based on preliminary investigations of irradiated volumes, we classified highly irregular shapes as RI $<0.6$. Following a similar approach for number of targets, we define an adjustment factor for shape regularity as follows:

$$
R(P)=\frac{a_{R I<0.6}(P)}{a_{R I>0.6}(P)}
$$

where the $a_{R I>0.6}(P)$ is the fit parameter for regularlyshaped targets. We can then express irradiated volume for complex shapes as irradiated volume of regular shapes times a scalar:

$$
V_{R I<0.6}(P)=R(P) \times V_{\text {primary }}(P)
$$

where $V_{\text {primary }}(P)$ is given by Eq. 1 . Note: $R(P)>=1$.

\section{Combined framework for irradiated volume}

For a given case involving $\mathrm{N}$ targets, of shape complexity RI, planned with a given technique, the combined framework defines the irradiated volume receiving any isodose line P as:

$$
V(P)=R(P) \times F_{N}(P) \times V_{\text {primary }}(P)
$$

where the factors $R$, and $F_{N}$ are used to bring irradiated volume around a single, simple-shaped target to the appropriate shape complexity and number of targets $(\mathrm{N})$ respectively. An error term $-\delta(\mathrm{P})-$ is the PI95 around the model. Factors $\mathrm{R}, \mathrm{F}_{\mathrm{N}}$ and $\delta$ can thus be characterized as functions of $\mathrm{P}$ in order that EXT or BMP receiving any isodose line can be interpolated.

\section{Framework testing}

The framework was applied and tested to two models as follows.

(1) Single-Arc, Homogeneous (SAHO) HF-RT: 181 consecutive HF-RT cases between 2013 and 2014 were retrospectively accessed under our institutional Review Ethics Board (REB) approval as follows: 1 target $(N=135), 2$ targets $(N=29), 3$ targets $(N=7)$, and $>3$ targets $(N=10)$. Target volumes ranged from 2.3 to $84.6 \mathrm{~cm}^{3}$ with a median of $14.1 \mathrm{~cm}^{3}$. All HF-RT cases were treated on a Synergy "S" linac (Elekta AB, Stockholm, Sweden) equipped with a $4 \mathrm{~mm}$ leaf width MLC at isocentre [19]. Treatment planning was performed using the Pinnacle $^{3}$ treatment planning system (TPS) v9.0 or 9.2 (Philips Healthcare, Andover, USA) with SmartArc optimization for VMAT and using a dose calculation grid of $2 \mathrm{~mm}$. The gross tumor volume (GTV) in the case of intact metastases, and clinical target volume (CTV) in the case of surgical cavities, were contoured on volumetric T1 post-gadolinium MRI fused to the treatment planning CT, which is acquired at $1 \mathrm{~mm}$ slice spacing. For intact metastases, no additional expansion to the GTV was made for microscopic spread, therefore CTV = GTV. A PTV margin of $2 \mathrm{~mm}$ was applied around each CTV. The 
target objective was to cover greater than $98 \%$ of the PTV $(V 100>98 \%)$ with the prescribed dose. The maximum dose in the target was limited to $<130 \%$ of prescribed dose, and was typically $<120 \%$, therefore referred to as being relatively "homogeneous". A single full arc was used for each case. The prescription dose ranged from 20 to $35 \mathrm{~Gy}$ in five fractions. All parameters $-F_{N}(P), R(P), a(P), b(P)$ and $\delta(P)$ were determined for $P=0.5$ to $P=0.8$ for both EXT and BMP based on curve fitting.

(2)Four-Arc, Heterogenous (FAHE): A subset of 30 randomly-selected cases was re-planned using the published four-arc technique of Clark et al. and later improved upon by Thomas et al. [12, 20]. In this approach, the same target objective (V100 > 98\%) was used, but there were no explicit constraints set for the maximum target dose, which resulted in relatively more "heterogeneous" dose distributions (mean Dmax $=140 \%$, range: $127-158 \%$ of the prescribed dose) compared to our institutional protocol, but consistent with the published literature. This technique is commonly used for single-fraction stereotactic radiosurgery (SRS), but we recognized that institutions may translate the same technique over to the larger-target HF-RT cases, which motivated applying our framework to this technique.

\section{Validation and feasibility}

Our clinical SAHO model was validated against 23 consecutive prospective cases from our institution receiving HF-RT that were not part of the modeling process. The 23 validation cases consisted of singletarget $(N=16)$, 2-target $(N=3)$, 3-target $(N=3)$ and $>3(N=1)$ cases, as well as five cases within the single-target group that had an $\mathrm{IR}<0.6$. The mean total target volume in the validation set was $25.0 \mathrm{~cm}^{3}$ (range: $2.5 \mathrm{~cm}^{3}-58.6 \mathrm{~cm}^{3}$ ). For all cases, we used Eq. 7 to determine $\mathrm{V}_{\text {primary }}$ by dividing the measured $\mathrm{V}(\mathrm{P})$ by the appropriate values of $\mathrm{F}_{\mathrm{N}}(\mathrm{P})$ and $\mathrm{R}(\mathrm{P})$. We defined a successful prediction to be a given data point falling within PI95 of our model. Additionally, we investigated whether fits made to the validation data fell within the CI95 of our model, which would indicate that the ensemble of validation data and model were statistically equivalent.

As published SRS data tends to follow the FAHE planning approach, we also used our framework to demonstrate that we can feasibly compare irradiated volumes against five independent publications $[11,12,16,18,21]$. It is important to note that in Thomas et al. and Bohoudi et al., the reported V12Gy is equivalent to EXT-67 or BMP67 in our series, since $12 \mathrm{~Gy}$ is $67 \%$ of $18 \mathrm{~Gy}$, which was the prescribed dose used in those two studies. This further demonstrates the flexibility of our framework to apply to any isodose level irrespective of prescribed dose.

\section{Results \\ SAHO}

The median (inner 95th percentile range) of RTOG conformity index [22], Paddick conformity index [23], and homogeneity index for the 135 plans used in the SAHO model were 1.17 (1.05-1.35), 0.83 (0.73-0.91), and 1.16 (1.08-1.23), respectively. The SAHO model is shown in Fig. 1 as EXT and BMP volumes (i.e. $\mathrm{V}_{\text {primary }}(\mathrm{P})$ as per Eq. 1) receiving doses from 50 to $80 \%$ in $10 \%$ increments versus target volume. The best-fit curves shown in Fig. 1 were used to obtain coefficients $a(P)$ and $b(P)$ as per Eq. 1. Spearman's correlation coefficients $\left(\mathrm{R}^{2}\right)$ were larger than 0.98 for the EXT volumes and in the range of $0.76-0.77$ for the BMP volumes, indicating a greater degree of spread in the data for BMP fits. The dashed lines indicated the upper and lower bounds of the PI95 while the dotted lines indicated the CI95.

\section{Number of targets shape regularity and application of FAHE}

The derivation of $\mathrm{F}_{\mathrm{N}}$ and $\mathrm{R}$ is illustrated at the $50 \%$ isodose level $(P=0.5)$ in Fig. 2 , parts (a) through $(\mathrm{c})$. Note that the equations shown are those from Fig. 1, plus an additional factor that corresponds to the $F_{N}$ or $R$ through curve-fitting and application of Eqs. (2) and (5), respectively.

As shown in Fig. $2 \mathrm{~d}$ at the $60 \%$ isodose level, our FAHE model generally resulted in lower irradiated volumes for any given target volume than our clinical SAHO model. Although not shown in Fig. 2d, FAHE treatment plans consisted of tighter MLC margins around the targets and, as a result of allowing for greater hotspots, greater Dmax relative to the prescribed dose (mean Dmax $=140 \%$, range: $127-158 \%$ of the prescribed dose).

Putting it all together, each term encompassed by Eqs. 1, 2 and 5, as well as the PI95 (termed $\delta$ ) are plotted in Fig. 3 as a function of P. As seen in Fig. 3(a) and (b), $\mathrm{a}(\mathrm{P})$ lowers for increasing values of $\mathrm{P}$, while the power term $b(\mathrm{P})$ remains relatively flat or modestly increases for BMP and EXT respectively. The derived values of $\mathrm{F}_{\mathrm{N}}, \mathrm{R}$ as per Eqs. (2) and (5) are plotted as a function of $\mathrm{P}$ in Fig. 3d through $\mathrm{f}$. As seen in Fig. 3(d) through (f), the correction for number of targets $(\mathrm{N})$ in this series is larger than for shape irregularity, with $\mathrm{F}_{\mathrm{N}}$ approaching a factor of 2 or 3 for $N>3$ targets in EXT or BMP respectively, while $\mathrm{R}$ has a plateau around 1.17 . The PI95 (or $\delta$ ) is higher for BMP than for EXT at all values of $\mathrm{P}$, 

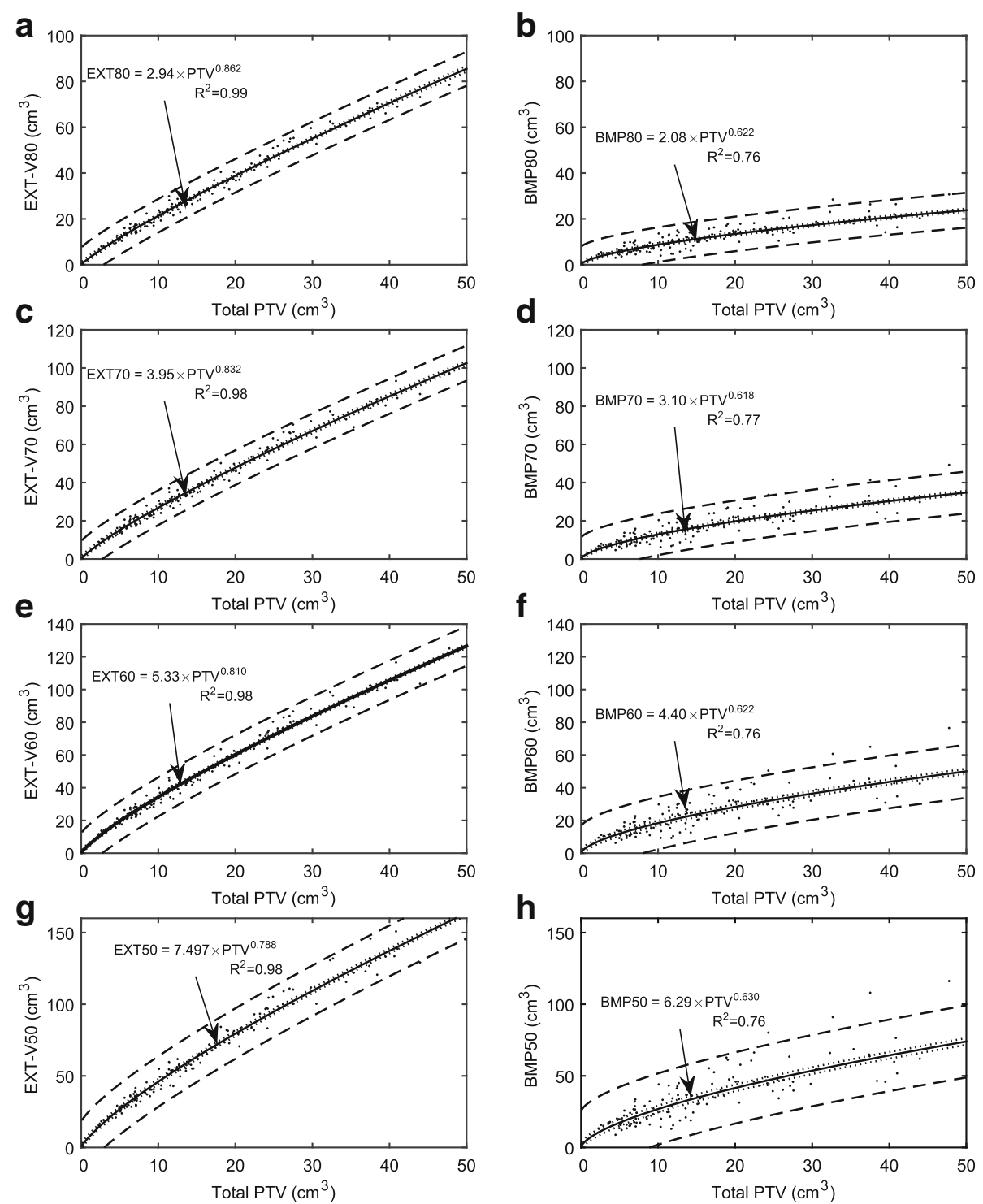

Fig. 1 Plots irradiated volume versus target volume using SAHO for single-target data. Left column $(\mathbf{a}, \mathbf{c}, \mathbf{e}$, and $\mathbf{g})$ are plots for the External (EXT) contour. Right column (b, d, $\mathbf{f}, \mathbf{h}$ ) are plots for the Brain-Minus-PTV (BMP) contour. Top down to bottom row: shows model for $80 \%, 70 \%, 60 \%$ and50\% of the prescription dose. Each dot represents one patient datum point. The best-curve equations according to Eq. (1) (for single-target data) are shown on the graphs. The dashed and dotted lines indicated the $95 \%$ prediction and confidence bands, respectively

as shown Fig. 3c, which can also be appreciated by the span of the dashed lines in Fig. 1.

\section{Validation and feasibility}

\section{SAHO}

As shown in Fig. 4, at both the 80 and 50\% isodose level, all of the data points were encompassed by the model's PI95 (dashed lines). Furthermore, best fit lines to the validation data for EXT-80 and EXT-50 were observed to fall within the model's CI95 (dotted lines), indicating that the model and validation data were not statistically different $(p>0.2)$. For BMP-80 and BMP-50, the best fit lines to the validation data fell below the CI95 but were still determined to be statistically equivalent when applying a Student T-test analysis $(p>0.2)$.

\section{FAHE}

As shown in Fig. 5, the PI95 (dashed lines) of our FAHE model encompassed all but one individual data point across five independent publications [11, 12, 16, 18, 21]. The one point that fell above the PI95 was actually generated using a single-arc technique thus one would expect a somewhat higher irradiated volume, closer to SAHO. Furthermore, irradiated volumes V67 and BMP67 from two selected publications, were encompassed nearly entirely by the narrow CI95 (dotted lines) 

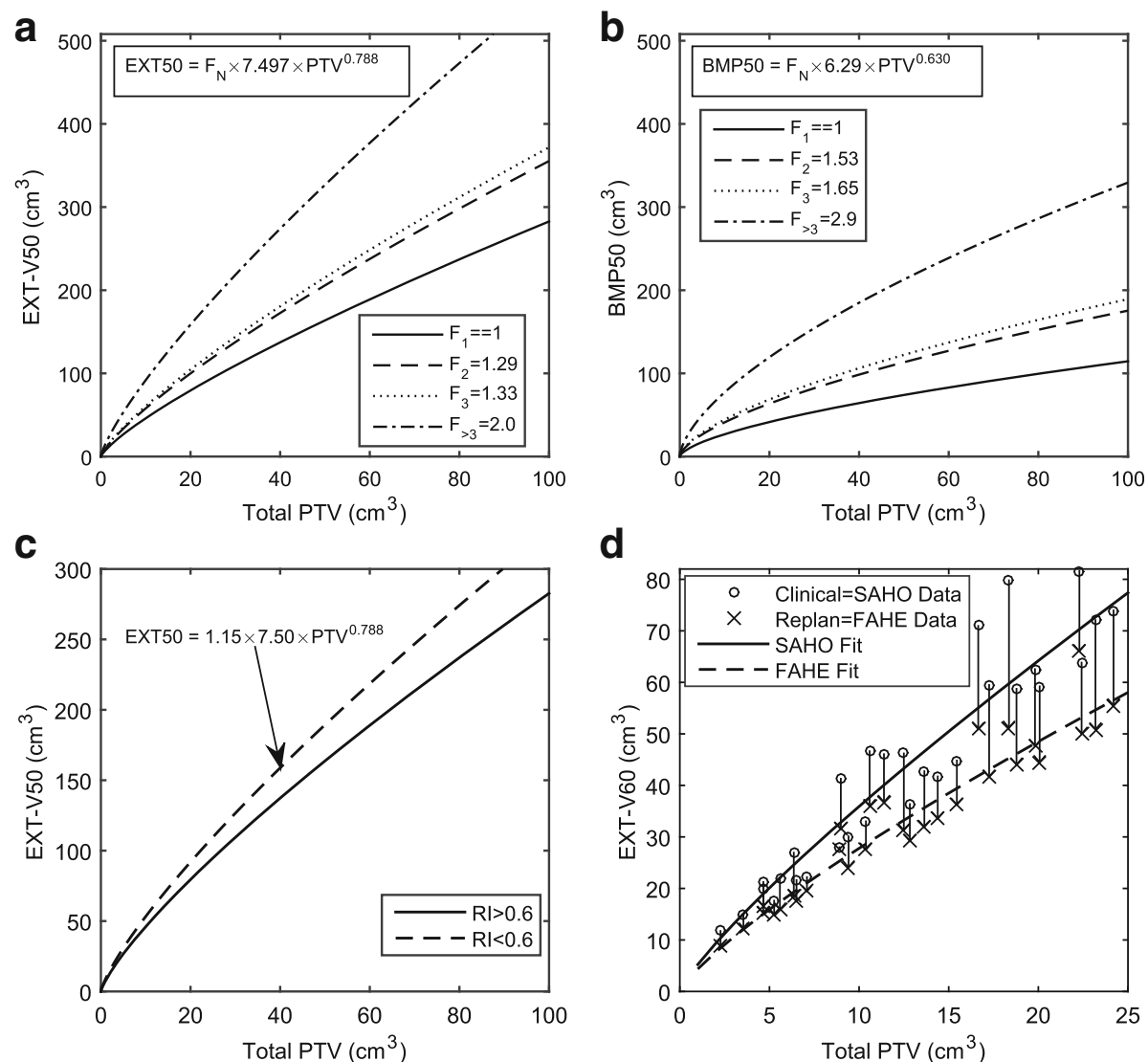

d

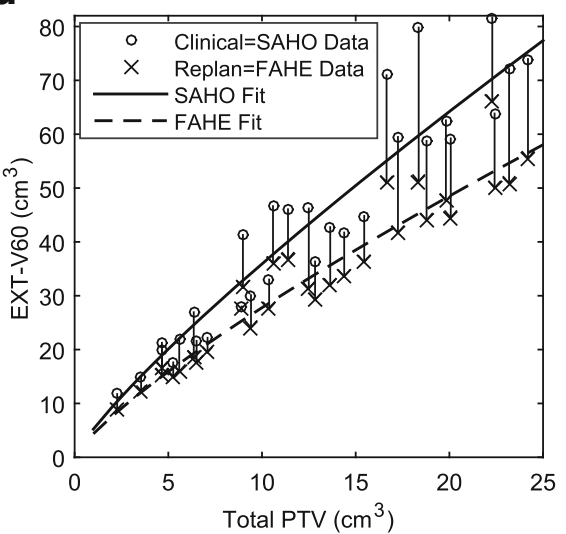

Fig. 2 Investigation of number of targets, shape regularity and treatment technique. $\mathbf{a}$ and $\mathbf{b}$ are EXT-50 and BMP-50, respectively, versus total PTV for plans with 1 target (primary model) compared to plans with 2, 3, and $>3$ targets for SAHO. The first term in the equations is the factor $\mathrm{F}_{\mathrm{N}}$ by which the primary SAHO model is multiplied by to achieve the indicated curve. Individual data points are not shown for ease of interpretation. c EXT-50 versus PTV for plans with RI > 0.6 compared to plans with target $\mathrm{RI}<0.6$. d EXT-60 vs Total PTV for a subset of clinical plans re-planned using FAHE. The arrows point from the original SAHO plan to the re-plan FAHE plan

of the FAHE model, indicating FAHE was statistically equivalent to the cited publications $[12,16]$. These results indicated that our framework can feasibly be applied across different publications and institutions as a means of comparison.

\section{Discussion}

We have developed and tested a readily accessible framework for irradiated volume in HF-RT. The framework describes irradiated volume of the external contour (EXT) and Brain-Minus-PTV (BMP) for any given target volume and isodose level that any institution can implement for their technique and patient population. The purpose is not to seek out which technique is "better" or "worse", but to simply facilitate a means for technique comparison.

The primary gain of the proposed framework is that despite the complex underlying physics behind it, irradiated volume can be reduced to a simple set of variables, which any institution can measure for their HF-RT patient population. Although we have limited the variables to target size, number, and shape for a given technique one could easily expand upon the model by adding other refining features, including MLC size, MLC margin around the target and number of arcs or isocenters. The clinical benefits of such a system include: (1) a framework through which institutions can perform treatment plan quality assurance, especially for complex situations such as HF-RT for which guidelines are lacking; (2) a framework through which treatment plans can be compared across institutions, which is often hampered by differing definitions of quality metrics. There are numerous published papers regarding the dosimetric benefit of one treatment technique over another, but the conclusions are often mixed, with some papers claiming little difference [12] and others citing larger differences [11]. The proposed framework may provide a means through which irradiated volume is parameterized and compared across institutions in a fair way. (3) a framework through which institutions can improve upon plan quality by reducing the variation in their own measured model over time. In our clinic, we have initiated using the proposed 


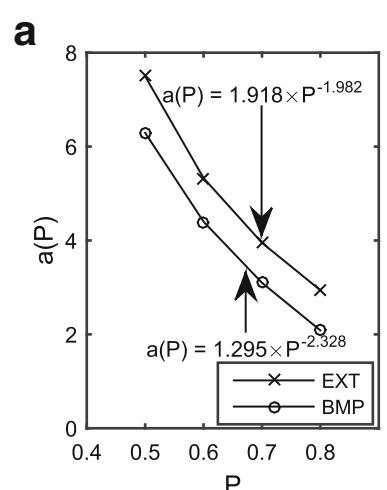

d

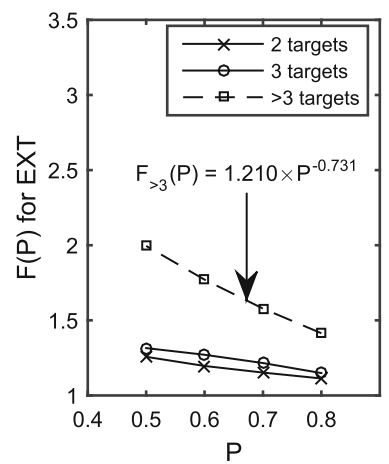

b

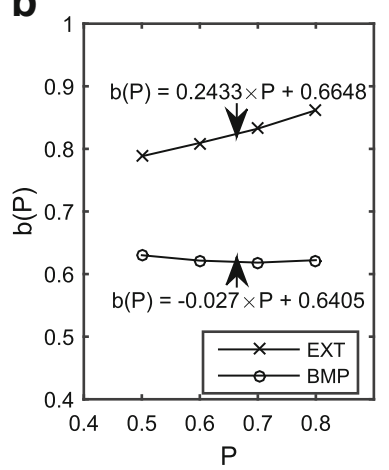

e

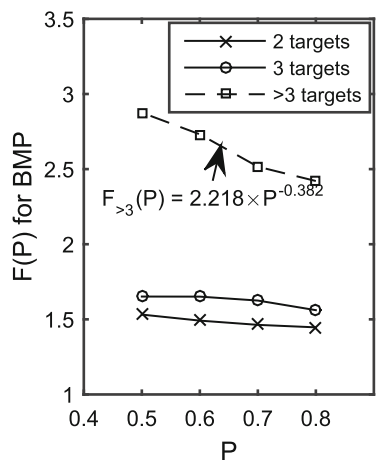

C

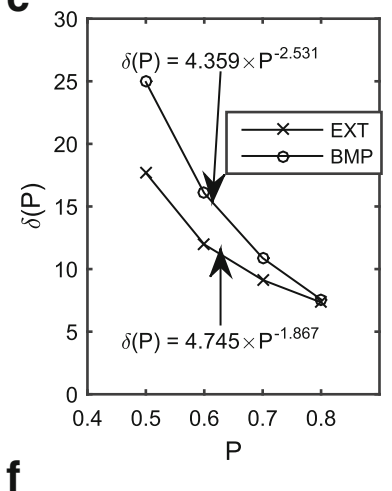

$\mathbf{f}$

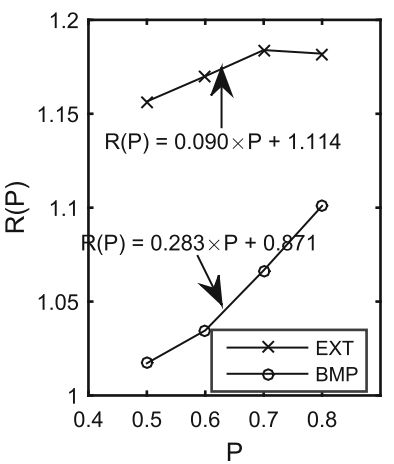

Fig. 3 Curve fitting parameters for parameterization of irradiated volume based on the SAHO technique. $\mathbf{a}$ and $\mathbf{b}$ show fit parameters $\mathbf{a}$ and $\mathbf{b}$ respectively for the primary model as in Eq. (1) and Fig. 1. $\mathbf{c}$ is the error $(\delta)$ equal to the $95 \%$ prediction interval. $\mathbf{d}$ and $\mathbf{e}$ show the factor $\mathrm{F}_{\mathrm{N}}(\mathrm{P})$ for EXT and BMP respectively. $\mathbf{f}$ shows the factor $\mathrm{R}(\mathrm{P})$

framework during the treatment plan review process by comparing irradiated volume parameters for upcoming plans against the model-predicted values. If a plan exceeds the prediction interval of the model, an investigation is undertaken to determine whether plan improvement can be made, and over time the spread of dosimetric data will diminish. Note that the prediction interval used in the present study was taken to be $95 \%$, which was selected to cover an accurate range of dosimetric outcomes, at the cost of having wider intervals than using a less-predictive model such as with an $80 \%$ prediction interval.

As an example, a centre relatively new to HF-RT wishes to treat an inoperable and relatively large intact brain metastasis that has a volume (after PTV expansion) of $60 \mathrm{~cm}^{3}$ with a fractionation scheme of $30 \mathrm{~Gy}$ in five fractions, for which there is limited data on dosevolume constraints for normal tissue. The clinic is interested in the volume of tissue receiving $>21 \mathrm{~Gy}$ in five fractions, which for a 30 Gy prescription is $70 \%$ of the prescribe dose, i.e. V70. From Fig. 1(c) and (d), if using a SAHO technique one may expect a range of V70 to be $119 \mathrm{~cm}^{3} \pm 9 \mathrm{~cm}^{3}$ or $39 \mathrm{~cm}^{3} \pm 11 \mathrm{~cm}^{3}$ for EXT and BMP respectively. If the clinic were willing to accept a hotter plan, with a target Dmax of up to $150 \%$ and planned with the FAHE technique, then the corresponding expected V70 would be lower than for SAHO (see Fig. 3d), in this case $83 \mathrm{~cm}^{3} \pm 6 \mathrm{~cm}^{3}$ and $27 \mathrm{~cm}^{3} \pm 8 \mathrm{~cm}^{3}$. If the target was irregularly shaped, or an additional target was also present, then the factors $F_{N}$ or $R$ could be appropriately applied. For any given isodose the same process can be applied, using the curves in Fig. 2 to extract the relevant parameters needed to asses irradiated volume. These ranges of irradiated volume serve as a guide of what may achievable: if a higher irradiated volume is achieved than the higher bound of the predicted range, then perhaps it may prompt a closer look at the complexity of the case or whether there can be improvements, whereas if a lower irradiated volume is achieved then that technique used is capable of achieving lower irradiated volumes, which may be a benefit.

An advantage of the present manuscript is that irradiated volume is described independently from prescription dose and PTV margin, and for both EXT and BMP, which facilitates comparison to published data. Certain treatment planning systems, such as Leksell GammaPlan, report irradiated volume to EXT whereas other planning systems can generate brain contours, and often subtract the PTV to define untreated brain, which we report as BMP. Irradiated BMP volumes are always less 

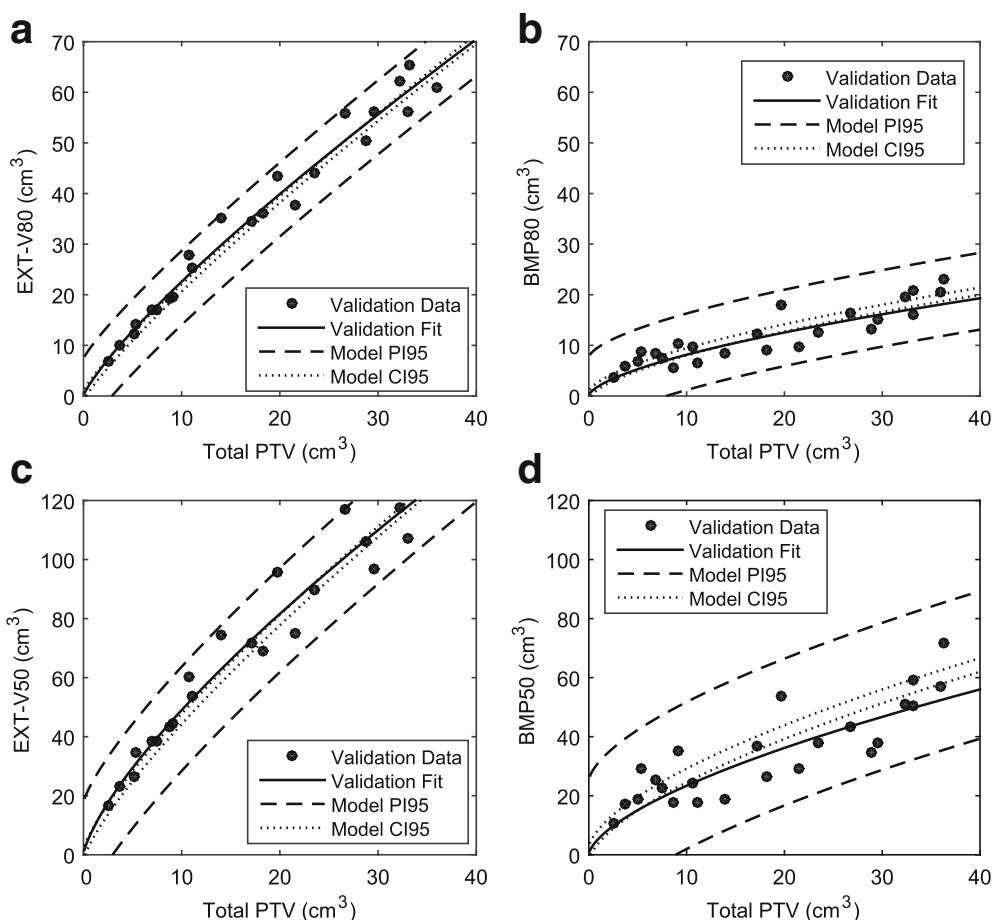

Fig. 4 Validation of the irradiated volume framework using SAHO against prospective data at our institution. Parts (a) and (b) are showing the irradiated volumes receiving $80 \%$ of the prescription for EXT and BMP, respectively. Parts (c) and (d) are showing the irradiated volumes receiving $50 \%$ of the prescription for EXT and BMP, respectively. The dashed lines are the upper and lower bounds of the 95\% prediction interval (P195) for the primary model. The dotted lines are the upper and lower bounds of the 95\% confidence interval (C195) for the SAHO model. EXT = External contour. BMP = BrainMinusPTV
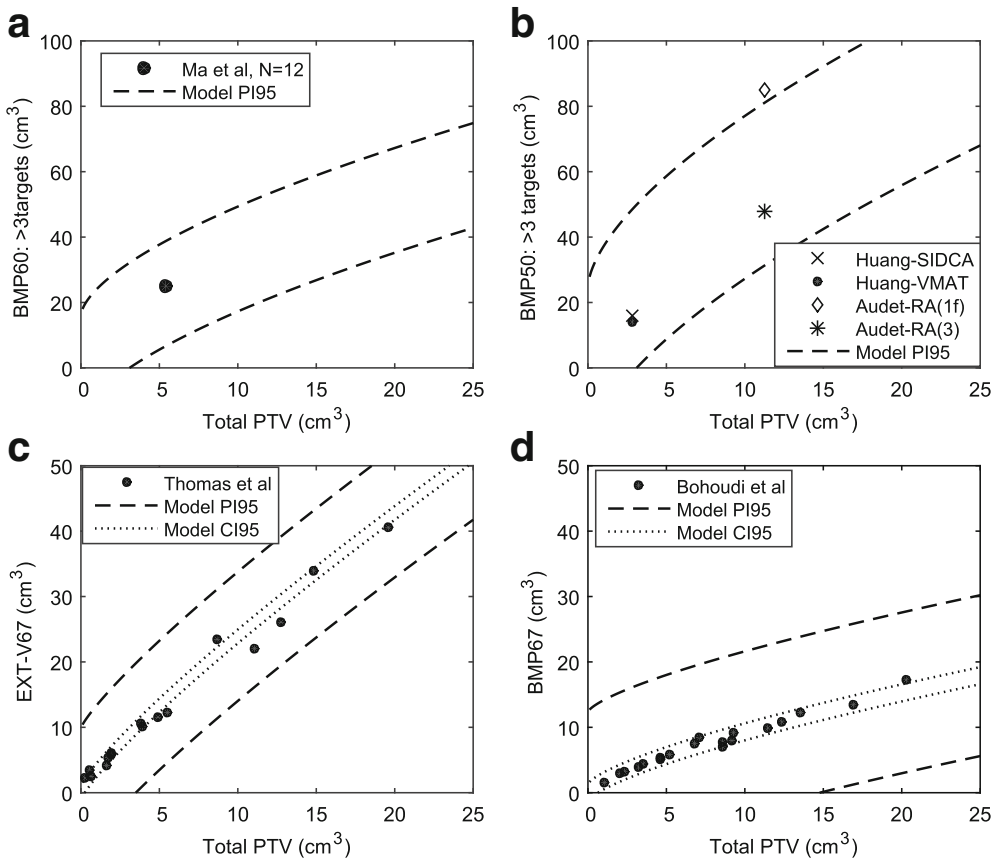

Fig. 5 Feasibility of applying irradiated volume framework to compare against published data. Each part corresponds to a particular metric found in the literature as follows: (a) is BMP60 for $>3$ targets; (b) is BMP50 for >3 targets; (c) is EXT-67 for single targets; (d) is BMP67 for single targets. The dashed lines are the upper and lower bounds of the 95\% prediction interval (PI95) for the FAHE model. The dotted lines (parts (c) and (d) only) are the upper and lower bounds of the 95\% confidence interval (CI95) for the FAHE model. EXT = External contour. BMP = BrainMinusPTV 
than EXT volumes (see Fig. 1), since the target and all dose spilling outside the brain contour are excluded from BMP. There is also more variation in the BMP curves: depending on the target location, moreor-less dose will spill beyond the brain into the skull and surrounding tissue. Our proposed framework also includes measuring EXT and BMP at multiple isodose levels such that any future isodose level can be interpolated, by presenting the fit parameters similarly as shown in Fig. 2.

Although we have demonstrated feasibility of our framework by comparing to internal and external data, there are certain limitations of the framework that need addressing. Firstly, our underlying clinical data is for single-arc HF-RT treatments. Although we re-planned 30 cases with a published FAHE technique, we do not use that approach clinically. Furthermore, our investigation of factors $F_{N}$ and $R$ may be limited to our treatment technique and patient population, which by virtue of being HF-RT, consisted of larger targets than those used for SRS. For example, our finding that having $>3$ targets resulted in higher (range: 1.4-2.9x) irradiated volumes than single-target cases may be due to increased dose interplay between targets resulting from the larger targets in our series (median and maximum volume $=14.1$ and $84.6 \mathrm{~cm}^{3}$ respectively) than for typical SRS series. By contrast, the single metastasis model proposed by Bohoudi et al. consisted of target volumes in the range of 1 to $20 \mathrm{~cm}^{3}$ (median $=7 \mathrm{~cm}^{3}$ ) and was validated against multiple targets [16]. Naturally, there are many factors affecting the extent of irradiated volume, including penumbra, MLC margin around the target, beam modulation and number of arcs. However, despite the limitations, reasonable agreement was found comparing our FAHE model to data from other institutions demonstrating feasibility for inter-institutional comparisons [12, 20, 21]. This shows that at a minimum the specific factors we found can be used as a starting point going forward, and refinements can be easily incorporated. It would be interesting to present irradiated volume data acquired from other systems, such as the Gamma Knife or Cyberknife, in a similar format to that in the present manuscript.

It is important to emphasize that there are other factors to consider than irradiated volume when evaluating treatment planning techniques. For example, the conformity of the prescription isodose line to the target can vary between techniques. Another factor to consider is dose homogeneity. For HF-RT, our institution has consistently employed a $2 \mathrm{~mm}$ PTV margin, which has limited the comfort of the treating physicians to allow hotspots in the target in excess of 120-130\%, as such steep gradients may cause normal-tissue necrosis within the margin itself. The dose limit of $130 \%$ is different from the situation of single-fraction SRS, in which the hotspot may easily extend up to $150-160 \%$. Furthermore, the number of arcs and the degree of modulation can affect dose fall-off. The treating centre should determine what maximum allowed dose and degree of modulation it is comfortable with. Other important plan quality metrics for dose fall-off include the Paddick Gradient Index (GI), which is the ratio of volume receiving half of the prescribed dose to the volume receiving the prescribed dose. While GI is easy to measure, the expectation of the present work is that that normal tissue tolerances for HF-RT will ultimately be derived from clinical studies and reported in absolute volumes, much like the safety limit of V12 $<10 \mathrm{~cm}^{3}$ is used for SRS. One of the major strengths of the present work is there are no assumptions made as to what an "optimal" plan is for any given case, since treatment plan optimality depends on a multitude of factors. Rather than attempt optimality, our approach is heuristic in nature, and uses plans delivered to patients that the practising physicians (many of who have greater than 10 years experience in the field) deemed clinically acceptable.

Among other study limitations is the lack of clinical outcomes associated with the reported irradiated volume data. Although outcomes modeling is outside the scope of this work inclusion would clearly strengthen impact, and this work is ongoing. Another study limitation is that relatively few SAHO cases had more than 2 or 3 targets; having more multitarget cases would increase the accuracy of the determined $F_{N}$. Although the framework can be extended to isodose lines $<50 \%, 50 \%$ represents a clinically relevant lower limit [12]. Furthermore, in choosing to make the present manuscript readily comparable to other papers in the literature, we assumed that relative isodose lines are maintained irrespective of the absolute prescription dose whereas the planning process must incorporate machine constraints such as gantry speed.

\section{Conclusions}

We have developed a framework for statistically describing irradiated volume in linac-based HF-RT. The model of irradiated volume we generated with clinical cases was validated against an independent internal dataset. We have also tested applying the framework to compare irradiated volume parameters against multiple published data. The framework is readily accessible to all institutions and independent of prescription dose or PTV margin. 


\section{Abbreviations}

BMP: Brain Minus PTV; sometimes referred to as "Normal" or untreated Brain in literature; C195: 95\% confidence interval; CTV: Clinical target volume; Dmax: Maximum dose; EXT: External contour (entire volume within external contour as defined on (T); FAHE: Four-arc Heterogeneous; $F_{\mathrm{N}}$ : Factor accounting for number (N) of targets; GTV: Gross tumor volume; Gy: Gray; HF-RT: Hypo-fractionated radiotherapy; MLC: Multi-leaf Collimator; MRI: Magnetic Resonance Imaging; P: Percentage of Prescription. Refers to specific isodose levels in percent; PI95: 95\% prediction interval; PTV: Planning Target Volume; R: Factor accounting shape regularity; Rl: Regularity index; RTOG: Radiation Therapy Oncology Group; SAHO: Single-arc Homogeneous: SRS: Stereotactic radiosurgery; TPS: Treatment planning system; TV: Target volume; V12: Volume receiving 12 Gy or greater; VMAT: Volumetric Modulated Arc Therapy

\section{Acknowledgements}

The authors are grateful to Dr. Evan Thomas for his insight and willingness to share data. The authors are also grateful to Dr. Miguel Palacios and Dr. Frank Lagerwaard for their willingness to share data.

\section{Funding}

Not applicable (no funding for current research).

\section{Availability of data and materials}

All data generated or analysed during this study are included in this published article.

\section{Authors' contributions}

MR collected data and was primary contributor to writing manuscript. ASAH, SM, HS, and MT provided clinical input to the manuscript. YL and ASAR were major contributors in writing the manuscript. BC, ASAR, and CY assisted in collecting data and interpreting the data. AK provided statistical support and statistical analysis. All authors read and approved the final manuscript.

\section{Ethics approval and consent to participate}

Retrospective data for the present study was obtained under internal Review Ethics Board (REB) approval.

\section{Consent for publication}

Not applicable.

\section{Competing interests}

The authors declare that they have no competing interests.

\section{Publisher's Note}

Springer Nature remains neutral with regard to jurisdictional claims in published maps and institutional affiliations.

\section{Author details}

'Department of Radiation Oncology, Sunnybrook Health Sciences Centre, Toronto, ON, Canada. ${ }^{2}$ Department of Radiation Oncology, University of Toronto, Toronto, ON, Canada. ${ }^{3}$ Research Design and Biostatistics, Sunnybrook Health Sciences Centre, Toronto, ON, Canada.

\section{Received: 3 February 2017 Accepted: 6 July 2017} Published online: 14 July 2017

\section{References}

1. Yamamoto M, Serizawa T, Shuto T, Akabane A, Higuchi Y, Kawagishi J, et al. Stereotactic radiosurgery for patients with multiple brain metastases (JLGK0901): a multi-institutional prospective observational study. Lancet Oncol. 2014;15:387-95.

2. Sahgal A, Aoyama H, Kocher M, Neupane B, Collette S, Tago M, et al. Phase 3 trials of stereotactic radiosurgery with or without wholebrain radiation therapy for 1 to 4 brain metastases: individual patient data meta-analysis. Int J Radiat Oncol Biol Phys. 2015;91: 710-7.

3. Minniti G, Scaringi C, Paolini S, Lanzetta G, Romano A, Cicone F, et al. SingleFraction Versus Multifraction ( $3 \times 9$ Gy) Stereotactic Radiosurgery for Large $(>2$ cm) Brain Metastases: A Comparative Analysis of Local Control and Risk of Radiation-Induced Brain Necrosis. Int J Radiat Oncol Biol Phys. 2016;95:1142-8.

4. Al-Omair A, Soliman H, Xu W, Karotki A, Mainprize T, Phan N, et al. Hypofractionated stereotactic radiotherapy in five daily fractions for postoperative surgical cavities in brain metastases patients with and without prior whole brain radiation. Technol Cancer Res Treat. 2013;12:493-9.

5. Ernst-Stecken A, Ganslandt O, Lambrecht U, Sauer R, Grabenbauer G. Phase II trial of hypofractionated stereotactic radiotherapy for brain metastases: results and toxicity. Radiother Oncol. 2006;81:18-24.

6. Manning MA, Cardinale RM, Benedict SH, Kavanagh BD, Zwicker RD, Amir C, et al. Hypofractionated stereotactic radiotherapy as an alternative to radiosurgery for the treatment of patients with brain metastases. Int J Radiat Oncol Biol Phys. 2000;47:603-8.

7. Khan L, Soliman H, Xu W, Ruschin ME, Phan N, Lochray F, et al. Outcomes With Hypofractionated Stereotactic Radiation Therapy (hfSRT) in Patients With Intact Brain Metastases and Postoperative Surgical Cavities. Int J Radiat Oncol Biol Phys. 2014;90:5318.

8. Lawrence YR, Li XA, el Naqa I, Hahn CA, Marks LB, Merchant TE, et al. Radiation dose-volume effects in the brain. Int J Radiat Oncol Biol Phys. 2010;76:S20-7.

9. Blonigen BJ, Steinmetz RD, Levin L, Lamba MA, Warnick RE, Breneman JC. Irradiated volume as a predictor of brain radionecrosis after linear accelerator stereotactic radiosurgery. Int J Radiat Oncol Biol Phys. 2010;77:996-1001.

10. Korytko T, Radivoyevitch T, Colussi V, Wessels BW, Pillai K, Maciunas RJ, et al. 12 Gy gamma knife radiosurgical volume is a predictor for radiation necrosis in non-AVM intracranial tumors. Int J Radiat Oncol Biol Phys. 2006;64:419-24.

11. Ma L, Petti P, Wang B, Descovich M, Chuang C, Barani IJ, et al. Apparatus dependence of normal brain tissue dose in stereotactic radiosurgery for multiple brain metastases. J Neurosurg. 2011;114:1580-4.

12. Thomas EM, Popple RA, Wu X, Clark GM, Markert JM, Guthrie BL, et al. Comparison of plan quality and delivery time between volumetric arc therapy (RapidArc) and Gamma Knife radiosurgery for multiple cranial metastases. Neurosurgery. 2014;75:409-17. discussion 17-8

13. Hossain S, Keeling V, Hildebrand K, Ahmad S, Larson DA, Sahgal A, et al. Normal Brain Sparing With Increasing Number of Beams and Isocenters in Volumetric-Modulated Arc Beam Radiosurgery of Multiple Brain Metastases. Technol Cancer Res Treat. 2016:15:766-71.

14. Shiraishi S, Tan J, Olsen LA, Moore KL. Knowledge-based prediction of plan quality metrics in intracranial stereotactic radiosurgery. Med Phys. 2015:42:908.

15. Narayanasamy G, Smith A, Van Meter E, McGarry R, Molloy JA. Total target volume is a better predictor of whole brain dose from gamma stereotactic radiosurgery than the number, shape, or location of the lesions. Med Phys. 2013:40:091714.

16. Bohoudi O, Bruynzeel AM, Lagerwaard FJ, Cuijpers JP, Slotman BJ, Palacios MA. Isotoxic radiosurgery planning for brain metastases. Radiother Oncol. 2016;120:253-57.

17. Ruschin M, Lee Y, Beachey D, Yeboah C, Wronski M, Babic S, et al. Investigation of Dose Falloff for Intact Brain Metastases and Surgical Cavities Using Hypofractionated Volumetric Modulated Arc Radiotherapy. Technol Cancer Res Treat. 2016;15:130-38.

18. Audet C, Poffenbarger BA, Chang P, Jackson PS, Lundahl RE, Ryu SI, et al. Evaluation of volumetric modulated arc therapy for cranial radiosurgery using multiple noncoplanar arcs. Med Phys. 2011;38:5863-72.

19. Patel I, Glendinning AG, Kirby MC. Dosimetric characteristics of the Elekta Beam Modulator. Phys Med Biol. 2005:50:5479-92.

20. Clark GM, Popple RA, Young PE, Fiveash JB. Feasibility of single-isocenter volumetric modulated arc radiosurgery for treatment of multiple brain metastases. Int J Radiat Oncol Biol Phys. 2010;76:296-302.

21. Huang $Y$, Chin K, Robbins JR, Kim J, Li H, Amro H, et al. Radiosurgery of multiple brain metastases with single-isocenter dynamic conformal arcs (SIDCA). Radiother Oncol. 2014;112:128-32.

22. Shaw E, Kline R, Gillin M, Souhami L, Hirschfeld A, Dinapoli R, et al. Radiation Therapy Oncology Group: radiosurgery quality assurance guidelines. Int J Radiat Oncol Biol Phys. 1993;27:1231-9.

23. Paddick I. A simple scoring ratio to index the conformity of radiosurgical treatment plans. Technical note. J Neurosurg. 2000:93(Suppl 3):219-22. 